\title{
A multicentre evaluation of two intensive care unit triage protocols for use in an influenza pandemic
}

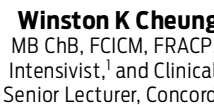
Clinical School ${ }^{2}$

John Myburgh

MB BCh, PhD, FCICM

Professor of Critical Care Medicine, St George Clinical School, ${ }^{3}$ and Director, Division of Critica Care and Trauma

Ian M Seppelt MB BS, FCICM, FANZCA Intensive Care Physician and Clinical Senio Lecturer $^{2}$

Michael J Par FRCP, FANZCA, FCICM Director of Intensive Care, and Associate Professor

Nikki Blackwell MB BS, FCICM Intensive Care Physician and Associate Professor in Critical Care

Shannon DeMonte GradCertClinN(CritCare) Clinical Nurse

Kalpesh Gandh MB BS, MD, FCICM Intensive Care Physician

Larissa Hoyling MN(CritCare) Intensive Care Research Nurse

Priya Nai MB BS, FCICM Intensive Care Physician

Melissa Passe BN, GradCertNeurosci. Clinical Research Nurse ${ }^{11,1}$

Claire Reynolds ICU Research Coordinator ${ }^{10}$

Nicholas M Saunders MB BS, MRCP, FRCA Senior ICU Registrar, ${ }^{12}$ and Specialist Registrar in Anaesthetics ${ }^{13}$

Manoj K Saxena MB BChir, BSc, FCICM Intensive Care Physician ${ }^{14}$ and Honorary Fellow ${ }^{4}$

Govindasamy

Thanakrishnan FRCP, FRACP, FCICM Intensivist $^{\dagger}$

on behalf of the Influenz Pandemic ICU Triage (PPIT) Study Investigators

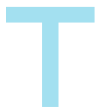

he concept of triage can be used to allocate medical resources in mass casualty events, such as a natural disaster, where the volume of patients presenting for medical attention exceeds the capacity to provide effective health care. ${ }^{1,2}$ Ethical issues surrounding the use of triage to prioritise patient management are challenging. ${ }^{1-3}$ Triage systems to allocate critical care resources have not yet been used in an influenza pandemic.

The outbreak of pandemic (H1N1) 2009 influenza required the provision of critical care services to large numbers of patients in Australia and New Zealand. ${ }^{4}$ In New South Wales, there were 519 intensive care unit (ICU) beds available to service a population of 7.2 million people. ${ }^{5,6}$ Equipment stockpiles could increase statewide ventilation capacity in a pandemic, but only by an additional $70 \% .^{5}$

Towards the end of the pandemic, in July 2009, the NSW Department of Health instituted a triage protocol to manage ICU resources during an influenza crisis when the surge in demand exceeded capacity by $50 \%$ (Box 1). ${ }^{5}$ The NSW triage protocol predominantly used criteria from a Minnesota protocol and added an element from a Canadian protocol, the Ontario Health Plan for an Influenza Pandemic (OHPIP, Box 2). 7,8

As the NSW triage protocol was instituted as a mandatory policy directive, ${ }^{5}$ our primary objective was to determine the increase in ICU bed availability that would result from its use. Our second objective was to compare this increase in bed availability with that from use of the OHPIP triage protocol.

\section{Methods}

\section{Setting and patients}

This prospective evaluation study was conducted in eight adult, general tertiary referral ICUs in NSW and

Objective: To determine the increase in intensive care unit (ICU) bed availability that would result from the use of the New South Wales and Ontario Health Plan for an Influenza Pandemic (OHPIP) triage protocols.

Design, setting and patients: Prospective evaluation study conducted in eight Australian, adult, general ICUs, between September 2009 and May 2010. All patients who were admitted to the ICU, excluding those who had elective surgery, were prospectively evaluated using the two triage protocols, simulating a pandemic situation. Both protocols were originally developed to determine which patients should be excluded from accessing ICU resources during an influenza pandemic.

Main outcome measure: Increase in ICU bed availability.

Results: At admission, the increases in ICU bed availability using Tiers 1, 2 and 3 of the NSW triage protocol were 3.5\%, $14.7 \%$ and $22.7 \%$, respectively, and $52.8 \%$ using the OHPIP triage protocol $(P<0.001)$. Re-evaluation of patients at 12 hours after admission using Tiers 1, 2 and 3 of the NSW triage protocol incrementally increased ICU bed availability by $19.2 \%, 16.1 \%$ and $14.1 \%$, respectively $(P<0.001)$. The maximal cumulative increases in ICU bed availability using Tiers 1, 2 and 3 of the NSW triage protocol were $23.7 \%, 31.6 \%$ and $37.5 \%$, respectively, at 72 hours $(P<0.001)$, and $65.0 \%$ using the OHPIP triage protocol, at 120 hours $(P<0.001)$.

Conclusion: Both triage protocols resulted in increases in ICU bed availability, but the OHPIP protocol provided the greatest increase overall. With the NSW triage protocol, ICU bed availability increased as the protocol was escalated.

Queensland, in the period after the 2009 influenza pandemic. Approval was obtained from the human research ethics committees of each institution, and the requirement for consent was waived.

All patients admitted to the ICUs were screened. Patients who had elective surgery were excluded, as it was expected that all elective surgery would be cancelled during an influenza pandemic. All remaining patients were evaluated by an ICU doctor using both the NSW and OHPIP triage protocols, to determine whether they qualified for admission to the ICU in a simulated pandemic situation. Patient admission and management were not actually altered by the study. The ICU experience of the assessing doctor varied between study centres, ranging from registrar to consultant level. After admission, the patients were re-evaluated using the NSW protocol at 12 and 72 hours, and the OHPIP protocol at 48 and 120 hours, to determine whether they should be discharged from the ICU.

Each ICU chose a continuous 6week observation period. Data were collected between September 2009 and May 2010.

\section{Triage protocols}

The triage protocols are described in Box 1 and Box 2. In brief, the NSW triage protocol had three tiers, which could be activated in a stepwise, timebased fashion as demand for critical care services increased. Tier 1 excluded patients from ICU admission if they had significant multiple organ failure, and discharged patients after 12 or 72 hours if they failed to respond to treatment. Tier 2 excluded patients with clinical conditions perceived to be associated with a poor prognosis, in addition to patients already excluded by Tier 1 . Tier 3 was designed to expand on Tier 2's list of clinical exclusion criteria, but at the time of the policy's introduction, only the Sequential Organ Failure Assessment (SOFA) scoring system from the OHPIP triage protocol had been incorporated. ${ }^{8,9}$

The OHPIP triage protocol had one tier and differed from the NSW protocol in its patient selection criteria. It only admitted patients who required mechanical ventilation or haemo- 


\section{New South Wales influenza pandemic triage protocol*}

Tier 1

Do not offer AND withdraw life-sustaining therapy from patients with any of the following:

1. Respiratory failure requiring intubation with persistent hypotension (systolic blood pressure $<90 \mathrm{mmHg}$ for adults) unresponsive to fluid therapy after $6-12$ hours and signs of additional end-organ dysfunction (eg, oliguria, decreased mental status, cardiac ischaemia)

2. Failure to respond to mechanical ventilation (no improvement in oxygenation or lung compliance) and antibiotics after 72 hours of treatment for a bacterial pathogen

3. Laboratory or clinical evidence of $\geqslant 4$ organ systems failing:

a. Pulmonary (acute respiratory distress syndrome, ventilatory failure, refractive hypoxia)

b. Cardiovascular (left ventricular failure, hypotension, new ischaemia)

c. Renal (hyperkalaemia, oliguria despite fluid resuscitation, increasing creatinine level) d. Hepatic (transaminase $>2$ times normal upper limit, increased bilirubin or ammonia levels)

e. Neurological (altered mental status not related to fluid volume status, metabolic or hypoxic source, stroke)

f. Haematological (clinical or laboratory evidence of disseminated intravascular coagulation)

g. Cirrhosis with ascites, history of variceal bleeding, fixed coagulopathy, or encephalopathy ${ }^{\dagger}$

h. Irreversible neurological impairment that makes the patient dependent for personal care (eg, severe stroke, congenital syndrome, persistent vegetative state) ${ }^{\dagger}$

Tier 2

Do not offer AND withdraw life-sustaining therapy from patients in respiratory failure requiring intubation with the following conditions, in addition to those in Tier 1. Patients with pre-existing system compromise or failure including:

1. Known congestive cardiac failure with ejection fraction $<25 \%$ (or persistent ischaemia unresponsive to therapy and pulmonary oedema)

2. Acute renal failure requiring haemodialysis

3. Severe chronic lung disease requiring home oxygen therapy ${ }^{\dagger}$

4. Immunodeficiency syndromes at a stage where the patient is susceptible to opportunistic pathogens

5. Active malignancy with poor potential for survival

6. Acute hepatic failure with hyperammonaemia

Tier 3

Specific triage protocols developed centrally and advised by specialist clinical groups:

1. Restriction of treatment based on disease-specific epidemiology and survival data for patient subgroups ${ }^{\dagger}$

2. Expansion of pre-existing disease classes that will not be offered ventilatory support 3. Applying SOFA scoring to the triage process, establishing a cut-off score ${ }^{\ddagger}$

SOFA = Sequential Organ Failure Assessment. * Reproduced with permission. ${ }^{5,7}$ The triage protocol applies to all patients undergoing assessment for possible critical care and not only those with influenza-like symptoms. Tier 1 is used initially; Tiers 2 and 3 can be sequentially activated later, as demand for intensive care unit resources escalates. $†$ Denotes criteria in the NSW triage protocol ${ }^{5}$ that were modified from the original triage criteria. $¥$ $\ddagger$ Refers to the prioritisation tool in the Ontario Health Plan for an Influenza Pandemic triage protocol (see Box 2).

dynamic support, on the basis that these forms of support fundamentally differentiate ICUs from other acute care areas. It excluded patients on the basis of advanced age and the requirement for resources that could not realistically be provided in a pandemic (eg, care for severe burns). Patients were also excluded at admission or discharged after 48 hours or 120 hours using criteria based on their SOFA score. ${ }^{8,9}$

\section{Outcome measures}

The primary outcome measure was increase in ICU bed availability, defined as the percentage of the total bed-days actually spent by patients in the ICU after the triage protocols had were followed up to discharge from or death in the ICU.

\section{Statistical analysis}

Descriptive statistics were calculated for all study variables. Continuous variables are reported as means and standard deviations. Increase in ICU bed availability was calculated as a percentage of the mean number of beds available per ICU per day ( \pm standard error). Bed availability results were compared using Pearson's $\chi^{2}$ test. Statistical analysis was performed by an independent statistician using Excel 2003 (Microsoft, Redmond, Wash, USA), SPSS version 17.0 (IBM, Armonk, NY, USA) and Confidence Interval Analysis (CIA) version 1.2 (BMJ Publishing Group, London, UK).

\section{Results}

Of 1262 patients admitted to the ICUs during the study period, 457 (36.2\%) were excluded because they were admitted after elective surgery. The remaining 805 patients $(63.8 \%)$ were evaluated using the NSW and OHPIP triage protocols. Complete data were collected for all patients.

There were 135 beds available for use in the eight ICUs during the study period (mean, $16.9 \pm 5.2$ beds per day per ICU). Demographic data of the patients are shown in Box 3. Patients had a mean length of stay in the ICU of $5.4 \pm 7.8$ days and occupied 4350 bed-days (76.7\% of 5670 total available bed-days). The overall ICU mortality rate was $11.7 \%$ (94 deaths).

Box 4 shows the cumulative increase in ICU bed availability resulting from evaluating patients using each tier of the NSW triage protocol at admission, 12 hours and 72 hours, and using the OHPIP protocol at admission, 48 hours and 120 hours. There were significant differences in the increase in ICU bed availability at admission between all tiers of the NSW triage protocol and the OHPIP protocol $(P<0.001)$.

Using the NSW triage protocol at admission, the increases in ICU bed availability using Tiers 1,2 and 3 were $3.5 \%, 14.7 \%$ and $22.7 \%$, respectively $(P<0.001)$. The incremental increases in ICU bed availability at 12 hours after admission using Tiers 1,2 and 3 were $19.2 \%, 16.1 \%$ and $14.1 \%$, respectively

$$
\begin{array}{r}
1 \text { Concord Repatriation } \\
\text { General Hospital, } \\
\text { Sydney, NSW. } \\
2 \text { University of Sydney, } \\
\text { Sydney, NSW. } \\
\text { 3 University of New South } \\
\text { Wales, Sydney, NSW. } \\
\mathbf{4} \text { The George Institute for } \\
\text { Global Health, } \\
\text { Sydney, NSW. } \\
\mathbf{5} \text { Nepean Hospital, } \\
\text { Sydney, NSW. } \\
\mathbf{6} \text { Liverpool Hospital, } \\
\text { Sydney, NSW. } \\
7 \text { Prince Charles Hospital, } \\
\text { Brisbane, QLD. } \\
\mathbf{8} \text { University of Queensland, } \\
\text { Brisbane, QLD. } \\
\mathbf{9} \text { Blacktown Hospital, } \\
\text { Sydney, NSW. } \\
10 \text { St Vincent's Hospital, } \\
\text { Sydney, NSW. } \\
11 \text { Ramsay Health Care, } \\
\text { Sydney, NSW. } \\
12 \text { Royal North Shore } \\
\text { Hospital, Sydney, NSW. } \\
13 \text { Norfolk and Norwich } \\
\text { University Hospital NHS } \\
\text { Foundation Trust, } \\
\text { Norwich, UK. } \\
\mathbf{1 4} \text { St George Hospital, } \\
\text { Sydney, NSW. } \\
\text { winston.cheung@ } \\
\text { sswahs.nsw.gov.au }
\end{array}
$$

MJA 2012; 197: 178-181 doi: 10.5694/mjall.10926 


\section{Ontario Health Plan for an Influenza Pandemic triage protocol*}

1. Assess whether the patient meets the inclusion criteria

- If yes, proceed to step 2

- If no, reassess patient later to determine whether clinical status has deteriorated

2. Assess whether the patient meets the exclusion criteria

- If no, proceed to step 3

- If yes, the patient is excluded from critical care ${ }^{\dagger}$

3. Proceed to prioritisation tool - initial assessment

Inclusion criteria

The patient must have one of the following:

A. Requirement for invasive ventilatory support

B. Hypotension with clinical evidence of shock refractory to fluid resuscitation, and requiring vasopressor or inotrope support

\section{Exclusion criteria}

The patient is excluded from admission or transfer to critical care if any of the following is present:

A. Severe trauma

B. Severe burns of patient with any two of the following: age $>60$ years; $>40 \%$ of total body surface area affected; inhalational injury

C. Cardiac arrest: unwitnessed cardiac arrest; witnessed cardiac arrest, not responsive to electrical therapy; recurrent cardiac arrest

D. Severe baseline cognitive impairment

E. Advanced untreatable neuromuscular disease

F. Metastatic malignant disease

G. Advanced and irreversible immunocompromise

$\mathrm{H}$. Severe and irreversible neurological event or condition

I. End-stage organ failure meeting the following criteria:

Heart

NYHA Class III or IV heart failure

Lungs

- COPD with $\mathrm{FEV}_{1}<25 \%$ predicted, baseline $\mathrm{PaO}_{2}<55 \mathrm{mmHg}$, or secondary pulmonary hypertension

Cystic fibrosis with postbronchodilator $\mathrm{FEV}_{1}<30 \%$ or baseline $\mathrm{PaO}_{2}<55 \mathrm{mmHg}$
- Pulmonary fibrosis with VC or TLC $<60 \%$ predicted, baseline $\mathrm{PaO}_{2}$ $<55 \mathrm{mmHg}$, or secondary pulmonary hypertension

- Primary pulmonary hypertension with NYHA Class III or IV heart failure, right atrial pressure $>10 \mathrm{mmHg}$, or mean pulmonary arterial pressure $>50 \mathrm{mmHg}$ Liver

- Child-Pugh score $\geqslant 7$

J. Age $>85$ years

$\mathrm{K}$. Elective palliative surgery

Prioritisation tool ${ }^{\ddagger}$

Criteria

\section{Action or priority}

Initial (admission) assessment

Exclusion criteria met or SOFA score $>11$ Exclude or discharge from critical care ${ }^{\dagger}$ SOFA score $\leqslant 7$ or single organ failure

Highest priority for access to critical care resources

SOFA score 8-11

Intermediate priority for access to critical care resources

No significant organ failure

Defer or discharge, reassess as needed

\section{8-hour assessment}

Exclusion criteria met or SOFA score $>11$ Discharge from critical care or SOFA score stable at 8-11 with no change

SOFA score $<11$ and decreasing

SOFA score stable at $<8$ with no change care resources

Intermediate priority for access to critical care resources

No longer dependent on ventilator

Discharge from critical care

120-hour assessment

Exclusion criteria met or SOFA score $>11$ Discharge from critical care or SOFA score $<8$ with no change

SOFA score $<11$ and decreasing progressively

Highest priority for access to critical care resources

SOFA score $<8$ with minimal decrease ( $<3$-point decrease in past 72 hours)

Intermediate priority for access to critical care resources

No longer dependent on ventilator

Discharge from critical care

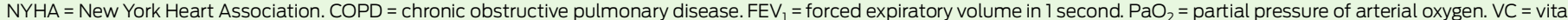
capacity. TLC = total lung capacity. SOFA = Sequential Organ Failure Assessment. ${ }^{9}$ ICU = intensive care unit.

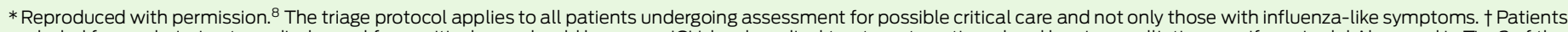

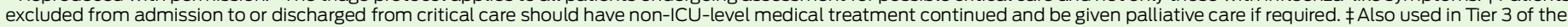
New South Wales triage protocol (see Box 1).

$(P<0.001)$. At 72 hours, there were further non-significant incremental increases in ICU bed availability of $0.9 \% \quad(P=0.42), 0.8 \% \quad(P=0.33)$ and $0.7 \%(P=0.26)$, respectively. The maximal cumulative increases in ICU bed availability for the three tiers were $23.7 \%, 31.6 \%$ and $37.5 \%$, respectively, at 72 hours $(P<0.001)$.

Using the OHPIP protocol at admission, the increase in ICU bed availability was $52.8 \%$. Using the protocol at 48 hours and 120 hours provided significant incremental increases in ICU bed availability of $6.7 \%$ and $5.4 \%$, respectively $(P<0.001)$. The cumulative increase in ICU bed availability at 48 hours was $59.5 \%$, with the maximal cumulative increase of $65.0 \%$ seen at 120 hours.
These figures indicate that a 17-bed general ICU would expect to make up to four beds available by using the NSW protocol at admission, depending on which tier was used, and about nine beds using the OHPIP protocol at admission. The NSW protocol would make an additional three beds available at 12 hours, but few additional beds thereafter. The OHPIP protocol would make one additional bed available at 2 days and another at 5 days.

\section{Discussion}

This prospective evaluation study, conducted soon after an influenza pandemic, found that the application of two triage protocols would increase ICU bed availability, with two distinct patterns.
Using the NSW triage protocol, all three tiers provided significant increases in ICU bed availability when patients were re-evaluated at 12 hours, but the maximal cumulative increase in ICU bed availability occurred with Tier 3. The OHPIP triage protocol provided the greatest increase in ICU bed availability overall, with a cumulative increase greater than any tier in the NSW triage protocol. Our results for the OHPIP protocol are similar to those from a retrospective study of two ICUs, which demonstrated that total ICU days would decrease by $52.6 \%$ with use of the protocol. ${ }^{12}$

The differences in ICU bed availability between the two protocols appear to be due to the medical acuity of the 
3 Baseline characteristics of 805 ICU patients*

\begin{tabular}{|c|c|}
\hline Age (years), mean $\pm S D$ & $60.4 \pm 19.4$ \\
\hline Male & $440(54.7 \%)$ \\
\hline $\begin{array}{l}\text { APACHE II score, } \\
\text { mean } \pm \text { SD }\end{array}$ & $18 \pm 8$ \\
\hline $\begin{array}{l}\text { Required mechanical } \\
\text { ventilation }\end{array}$ & $396(49.2 \%)$ \\
\hline \multicolumn{2}{|l|}{ Diagnosis at admission $^{\dagger}$} \\
\hline Medical $^{\ddagger}$ & $508(63.1 \%)$ \\
\hline Cardiovascular & $135(16.8 \%)$ \\
\hline Respiratory & $136(16.9 \%)$ \\
\hline Gastrointestinal & $44(5.5 \%)$ \\
\hline Neurological & $62(7.7 \%)$ \\
\hline Metabolic & 45 (5.6\%) \\
\hline Trauma & $52(6.5 \%)$ \\
\hline Head injury & $21(2.6 \%)$ \\
\hline Sepsis & $28(3.5 \%)$ \\
\hline Surgical $^{\S}$ & $219(27.2 \%)$ \\
\hline Cardiovascular & $29(3.6 \%)$ \\
\hline Gastrointestinal & $61(7.6 \%)$ \\
\hline Neurological & $41(5.1 \%)$ \\
\hline Respiratory & $57(7.1 \%)$ \\
\hline Trauma & $16(2.0 \%)$ \\
\hline Head injury & $7(0.9 \%)$ \\
\hline Undefined $^{\text {g }}$ & $78(9.7 \%)$ \\
\hline
\end{tabular}

$\mathrm{ICU}=$ intensive care unit. $\mathrm{APACHE}=$ Acute Physiology and Chronic Health Evaluation.

* Excludes those having elective surgery.

† Defined by the Australian and New Zealand

Intensive Care Society Centre for Outcome

and Resource Evaluation Adult Patient

Database.." $¥$ Patients admitted to the ICU

from a location other than an operating

theatre. \$ Patients admitted to the ICU from

an operating theatre. Я All patients with

diagnoses coded as undefined (eg,

"Cardiovascular - undefined").

patients excluded or discharged from the ICU. The NSW protocol focused on excluding patients with significant organ failures or comorbidities at admission. Fewer patients fulfilled the NSW exclusion criteria, resulting in smaller bed availability savings. The OHPIP protocol made more beds available early in the triage process by excluding the large proportion of patients who had a low risk of death.

The strength of our study was its multicentre, prospective design. The study ICUs were all general adult ICUs in Australian tertiary hospitals. Our findings are likely to be generalisable in Australia, and possibly in similar health systems, such as Canada.

While both triage protocols were limited by a lack of precise definitions for many of the exclusion criteria, these were taken at face value to examine how the protocols would perform in real-world clinical conditions. Interpretation of criteria was left to the discretion of the doctor administering the protocol, and it is likely that operator variability resulted in differences in interpretation, even with relatively simple conditions.

The major limitation of our study was that we conducted it after a pandemic had subsided, rather than during a pandemic. Many factors influence admission decisions during a mass casualty event. Evaluation of the protocols during an influenza pandemic may have given different results, as admission, discharge and treatment patterns may have changed. Only half of the patients in this study required mechanical ventilation, but normal admission criteria may become more stringent during a pandemic, resulting in a higher proportion of patients admitted to the ICU being mechanically ventilated.

The justification for excluding patients with high predicted mortality rates from ICU care in a triage protocol is that they are less likely to survive, and therefore less likely to have outcomes improved by ICU care. However, selective application of triage criteria may be considered ethically unjust if patients with similar ICU mortality rates are treated differently. ${ }^{1}$ It is therefore important that mortality rates for patients excluded by triage protocols are accurately determined.

Future studies should evaluate influenza pandemic triage protocols in real pandemic situations and determine whether they can be improved. Complex computerised simulation may represent an alternative, although less rigorous, evaluation method. ${ }^{13}$ Future studies should evaluate these protocols in different medical jurisdictions. Research also needs to determine whether such restrictive measures are acceptable to the communities in which they will be used in a public health crisis.

In conclusion, when tested in a noninfluenza pandemic setting, both the NSW and OHPIP triage protocols provided increases in ICU bed availability, but the OHPIP protocol provided the greatest increase overall. Using the NSW triage protocol, ICU bed availability increased as the protocol was escalated.
4 Cumulative increase in ICU bed availability using the two triage protocols*

$\checkmark$ OHPIP triage protocol $\quad \square$ NSW triage protocol Tier 2 $\rightarrow-$ NSW triage protocol Tier $3 \quad \square$ NSW triage protocol Tier $]$

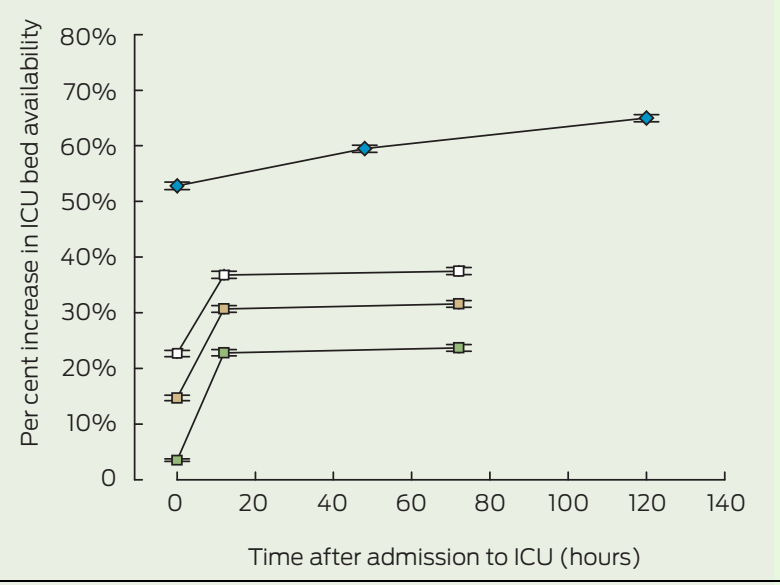

$\mathrm{ICU}=$ intensive care unit. OHPIP = Ontario Health Plan for an Influenza Pandemic. NSW = New South Wales. * Cumulative percentage increase in ICU bed availability for each evaluation time point in the triage protocols. Bars indicate standard error.

Acknowledgements: We thank Jennifer Peat, Concord Repatriation General Hospital, for her help with the statistical analysis, and the George Institute NSW ICU Research Group. We are indebted to the other investigators and participating hospitals - Blacktown Hospital: Dhaval Ghelani, Kiran Nand, Treena Sara; Concord Repatriation General Hospital: Helen Wong; Liverpool Hospital: Sharon Micallef; Prince Charles Hospital: Daniel Mullany; Royal North Shore Hospital: Simon Bird, Jane Bowey, Anthony Delaney; St George Hospital: Deborah Inskip, Rebecca Sidoli; Nepean Hospital and St Vincent's Hospital - and the dedicated medical and nursing staff at all the hospitals for their help with this study. This study was funded solely by the participating ICUs.

Competing interests: No relevant disclosures.

Received 19 Jul 2011, accepted 17 Feb 2012.

1 Sztajnkrycer MD, Madsen BE, Báez AA. Unstable ethical plateaus and disaster triage. Emerg Med Clin North Am 2006; 24: 749-768.

2 White DB, Katz MH, Luce JM, Lo B. Who should receive life support during a public health emergency? Using ethical principles to improve allocation decisions. Ann Intern Med 2009; 150: 132-138.

3 Kaposy C. Accounting for vulnerability to illness and social disadvantage in pandemic critical care triage. J Clin Ethics 2010; 21: 23-29.

4 ANZIC Influenza Investigators. Critical care services and 2009 HIN1 influenza in Australia and New Zealand. N Engl J Med 2009; 361: 1925-1934.

5 NSW Health. Influenza pandemic - providing critical care. Policy Directive PD2010_028. Sydney: NSW Health, 2010. http://www.health.nsw.gov.au/ policies/pd/2010/PD2010_028.html (accessed Nov 2010).

6 Australian Bureau of Statistics. Australian demographic statistics, Mar 2011. Canberra: ABS, 2011. (ABS Cat. No. 3101.0.) http://www.abs.gov.au/AUSSTATS/ abs@.nsf/DetailsPage/3101.0Mar\%202011?OpenDocument (accessed Feb 2012 ).

7 Hick JL, O'Laughlin DT. Concept of operations for triage of mechanical ventilation in an epidemic. Acad Emerg Med 2006; 13: 223-229.

8 Christian MD, Hawryluck L, Wax RS, et al. Development of a triage protocol for critical care during an influenza pandemic. CMAJ 2006; 175: 1377-1381.

9 Vincent JL, Moreno R, Takala J, et al; Working Group on Sepsis-Related Problems of the European Society of Intensive Care Medicine. The SOFA (Sepsis-related Organ Failure Assessment) score to describe organ dysfunction/failure. Intensive Care Med 1996; 22: 707-710.

10 Knaus WA, Draper EA, Wagner DP, Zimmerman JE. APACHE II: a severity of disease classification system. Crit Care Med 1985; 13: 818-829.

11 Australian and New Zealand Intensive Care Society Centre for Outcome and Resource Evaluation (CORE). CORE data collection tools. http:// www.anzics.com.au/core/data-collection-tools (accessed Mar 2011).

12 Christian MD, Hamielec C, Lazar NM, et al. A retrospective cohort pilot study to evaluate a triage tool for use in a pandemic. Crit Care 2009; 13: R170. doi: 10.1186/ сc8146.

13 Burton A, Altman DG, Royston P, Holder RL. The design of simulation studies in medical statistics. Stat Med 2006; 25: 4279-4292. doi: 10.1002/sim.2673. 
- Research

182 MJA $197(3) \cdot 6$ August 2012 